\title{
WEITERBILDUNG: HINWEISE ZUM FERNSTUDIUM DER BIOWISSENSCHAFTEN IN DER GRUND- UND SEKUNDARSTUFE
}

\section{ORIGINALER ARTIKEL}

DENDASCK, Carla Viana ${ }^{1}$, OLIVEIRA, Euzébio de ${ }^{2}$, FECURY, Amanda Alves ${ }^{3}$, DIAS, Claudio Alberto Gellis de Mattos ${ }^{4}$

DENDASCK, Carla Viana. Et al. Weiterbildung: Hinweise zum Fernstudium der Biowissenschaften in der Grund- und Sekundarstufe. Revista Científica Multidisciplinar Núcleo do Conhecimento. Jahrgang 06, Ed. 08, Vol. 06, S. 171-186. August 2021. ISSN: 2448-0959, Zugangslink: https://www.nucleodoconhecimento.com.br/bildung-de/biowissenschaften, DOI: 10.32749/nucleodoconhecimento.com.br/bildung-de/biowissenschaften

\section{ZUSAMMENFASSUNG}

Obwohl die Diskussionen über die Möglichkeit von Online-Bildung in der Grund- und Oberschule in Brasilien in den Zuge der theoretischen Debatte eingetreten sind, gibt es immer noch großen Widerstand. Der Pandemiekontext brachte jedoch keine Alternativen und führte Tausende von Studenten für mehr als ein Jahr in soziale Isolation. Dieser Kontext veranlasste Grund- und Oberschullehrer, sich an neue Technologien anzupassen, was die Notwendigkeit zeigte, sich auf die Arbeit im

\footnotetext{
${ }^{1}$ Theologe, PhD in Klinischer Psychoanalyse. Er arbeitet seit 15 Jahren mit Wissenschaftliche Methodik (Untersuchungsmethode) in der wissenschaftlichen Produktionsberatung von Master- und Doktoranden. Spezialist für Marktforschung und Forschung mit Schwerpunkt Gesundheit. Doktorand in Kommunikation und Semiotik (PUC SP).

${ }^{2}$ Biologe, PhD in Tropenkrankheiten, Professor und Forscher am Kurs für Leibeserziehung, Bundesuniversität Pará (UFPA).

${ }^{3}$ Biomedizin, PhD in Tropenkrankheiten, Professor und Forscher des Medizinischen Kurses des Macapá Campus, Bundesuniversität Amapá (UNIFAP), Prorektor für Forschung und Graduiertenstudien (PROPESPG) der Bundesuniversität Amapá (UNIFAP).

${ }^{4}$ Biologe, PhD in Theorie- und Verhaltensforschung, Professor und Forscher des Chemie-Studiengangs des Instituts für Grund-, Technik- und Technologiepädagogik (IFAP) und des Graduiertenprogramm in beruflicher und technologischer Bildung (PROFEPT IFAP).
}

RC: 96094

Verfügbar in: https://www.nucleodoconhecimento.com.br/bildung- 
Fernunterricht vorzubereiten. Die Leitfrage dieses Materials lautete: Wie können sich Life-Science-Lehrer vorbereiten und welche grundlegenden Werkzeuge sollten sie kennen, um ihren Online-Unterricht vorzubereiten? Daher bestand das allgemeine Ziel darin, Hinweise zu geben, die Lehrer kennen sollten, um ihren Online-Unterricht vorzubereiten. Die angewandte Methodik war explorativ durch Literaturrecherche. Die Hauptindikationen waren, dass Lehrer bei der Vorbereitung von Klassen nach diversifizierten Inhalten suchen müssen, um Motivationsstrategien mit ihren Schülern zu verfolgen und sich bei der Produktion von Klassen um das Thema Sprache, Audio und Licht zu kümmern. Schließlich liegt es am Lehrer, die verschiedenen Werkzeuge und Ressourcen wie Youtube, Instagram, Filme, Spiele und andere Subventionen zu erkennen, so dass es eine Annäherung zwischen dem Biologieunterricht und der Realität des Schülers gibt.

Schlüsselwörter: Lehrerausbildung, Fernunterricht, Biologieunterricht.

\section{EINLEITUNG}

Seit Ende 2019 stößt die Welt auf das SARS/COVID-19-Virus. Seine hohe Ansteckungsfähigkeit, die Beteiligung lebenswichtiger Organe, insbesondere der Lunge, und die Ignoranz der wissenschaftlichen Gemeinschaft veranlassten die Internationalen Organisationen, eine globale Pandemiesituation zu dekretieren, was auf einen sofortigen sozialen Rückzug hindeutet. Diese Maßnahme wirkte sich direkt auf Organisationen aus, die sich an neue Realitäten anpassen mussten, indem sie wie nie zuvor technologische Mittel einsetzten, um die Kontinuität ihrer jeweiligen Funktionalitäten zu ermöglichen. Nach Gonçalves et al. (2020) in Brasilien waren Schulen, insbesondere öffentliche Schulen, eine der am stärksten betroffenen Institutionen, da ihre Lehrer nicht nur mit Problemen in Bezug auf die Zugänglichkeit technologischer Werkzeuge konfrontiert waren, sondern auch nicht auf diese Realität vorbereitet waren und viele sich der Idee der Online-Bildung widersetzten, insbesondere in der Oberschule und Grundschule.

RC: 96094

Verfügbar in: https://www.nucleodoconhecimento.com.br/bildung- 
Lenzi und Santos (2021) rechneten damit an, dass es auch bei Impfstoffen und der Rückkehr zum Präsenzunterricht wichtig ist, dass sich die Lehrer an die Routinen des Fernunterrichts anpassen, insbesondere in dem, was von Lehrern als hybrider Unterricht befürwortet wurde, weil dieser Kontext bereits Realität ist. Aus diesem Grund soll diese Studie einige indikative Subventionen bereitstellen, die Lehrer bei der Vorbereitung ihrer Online-Kurse berücksichtigen sollten, beginnend mit der folgenden Leitfrage: Wie können sich Lehrer in den Biowissenschaften vorbereiten und welche grundlegenden Werkzeuge sollten sie kennen, um ihre Online-Kurse vorzubereiten? Daher wird das allgemeine Ziel dieser Studie sein: Hinweise zu geben, die Lehrer kennen sollten, um ihre Online-Kurse vorzubereiten.

Spezifische Ziele werden in Betracht gezogen: a) Aufzeigen der Hauptschwierigkeiten der Online-Ausbildung der Biowissenschaften und der Bedeutung des Einsatzes von Technologien; b) Nach Tools suchen, die in die Online-Bildung integriert werden können; c) Bereitstellung technologischer Indikatoren und Anweisungen, die vom Biologielehrer berücksichtigt werden sollten. So wurde in der theoretischen Grundlage eine breite Suche in Google Academic verwendet, die als Deskriptoren verwendet wurde: "Werkzeuge für den OnlineUnterricht der Biologie"; "Fernstudium Biologie"; "Hybride Lehre der Biowissenschaften"; "Biologie-Hybrid-Lehre". Als Einschlusskriterien wurden Materialien betrachtet, die zu dem hier vorgestellten Problem und den zielen beitragen könnten. Zwanzig Materialien wurden ausgewählt, darunter wissenschaftliche Artikel, Bücher und Handbücher. Im Besitz dieser wurde in den Ergebnissen und Diskussionen ein Tisch mit Hinweisen auf grundlegende Werkzeuge und allgemeine Überlegungen gehalten, die in der Lehre der OnlineBiologie zu berücksichtigen sind.

RC: 96094

Verfügbar in: https://www.nucleodoconhecimento.com.br/bildung- 


\section{DIE BEDEUTUNG DER LEHRERFORTBILDUNG}

Die Geschichte der Menschheit wird aus verschiedenen sozialen, politischen, wissenschaftlichen und wirtschaftlichen Perspektiven gelenkt und konstituiert, und all diese Dimensionen werden durch den technologischen Fortschritt beeinflusst (TREBIEN et al., 2020). Jede soziale und systemische Veränderung wirkt sich direkt auf den Bildungsprozess in der Schule aus, und daher muss die Schuleinrichtung diesen Fortschritten folgen (RODRIGUES; LIMA; VIANA, 2017). Die Schule muss sich an das neue Modell anpassen. Angesichts dieses Szenarios ist der Professor stark in einen Prozess involviert, der ständige Veränderungen impliziert, damit er den Herausforderungen der Gesellschaft begegnen kann. Denn wir leben im Informationszeitalter und somit ist die Erstausbildung von Lehrern keine ausreichende Voraussetzung mehr, um die Beherrschung von Inhalten und pädagogischer Qualität zu gewährleisten (JUNGES; KETZER; OLIVEIRA, 2018).

Die Weiterbildung nimmt die Aktivitäten auf, die von den Lehrern nach der ersten Ausbildung entwickelt wurden. Dies geschieht, wenn der Fachmann in den Lehrerberuf eintritt. Es kann individuell oder kollektiv sein (TREBIEN et al., 2020). Es wird daher unverzichtbar, ein Prozess der Bildung, der dauerhaft und kontinuierlich ist, verwurzelt in einer Erkenntnistheorie der Praxis und des täglichen Lebens, in der Lage zu sein, eine innovative und bewusste pädagogische Aktion zu subventionieren (JUNGES; KETZER; OLIVEIRA, 2018). Aufgrund der Relevanz der Kontinuität der Lehrerausbildung enthält das Gesetz über Richtlinien und Grundlagen der Bildung (LDB) ein Kapitel, das das Thema hervorhebt und diese Ausbildung unterstützt: "[...] Die Verbindung zwischen Theorien und Praktiken, auch durch berufsbegleitende Weiterbildung", im Anschluss an den einzigen Absatz, hebt die "Weiterbildung für Fachkräfte an ihrem Arbeitsplatz" hervor (TREBIEN et al., 2020, 94).

Angesichts der Zunahme und Verbesserung der Auf weiterbildungspolitischen Maßnahmen, vor allem aufgrund der Sorge um die Qualität der Lehrtätigkeit, hat das

RC: 96094

Verfügbar in: https://www.nucleodoconhecimento.com.br/bildung- 
Thema neue Maßnahmen aufgeworfen. Es ist dann notwendig, sich einem gut konzipierten Weiterbildungsprogramm mit gut strukturierten Zwecken anzuschließen, das nicht nur die Bedürfnisse des Lehrers, sondern auch das gesamte Bildungssystem umfasst und die Lehrer in diesen Planungsprozess einbetrifft, der partizipativ und kollaborativ sein und ihre tatsächlichen Bedürfnisse erkennen muss (RODRIGUES; LIMA; VIANA, 2017). Damit ihr Wissen und ihre Praxis geschätzt werden, muss der Lehrer zusammen mit seinen Gleichaltrigen reflektieren und miteinander in Dialog treten und anderen helfen, ihre Konflikte zu überwinden (FALSARELLA, 2004). Auf diese Weise trägt die Weiterbildung zur Entwicklung der Autonomie und Dynamik des Lehrers in seiner täglichen Praxis bei:

[...] Weiterbildung als beabsichtigter und geplanter Vorschlag, der darauf abzielt, den Erzieher durch einen reflexiven, kritischen und kreativen Prozess zu verändern, wird der Schluss gezogen, dass er den Lehrer motivieren sollte, ein aktiver Akteur in der Erforschung seiner eigenen pädagogischen Praxis zu sein, Wissen zu produzieren und in die Realität einzugreifen (FALSARELLA, 2004, S. 50).

Die Weiterbildung des Lehrers impliziert die Annahme einer kontinuierlichen Haltung, die Reflexion, Forschung, Aktion, Entdeckung, Organisation, Grundlage, Überprüfung und theoretische Konstruktion beinhaltet. Es geht nicht nur um das Erlernen neuer pädagogischer Techniken oder der neuesten pädagogischen Innovationen (FALSARELLA, 2004). In diesem Sinne muss der Prozess der permanenten Ausbildung auf der Realität jeder Schule basieren, und es ist notwendig, dass pädagogische Begegnungen und andere Momente der Ausbildung innerhalb und außerhalb der Schule jeden Tag neu überdacht werden, um den Veränderungen der Gesellschaft selbst zu folgen (GADOTTI, 2008). Die Rolle des Lehrers in diesem stark veränderlichen und dynamischen Kontext impliziert die Kontextualisierung der vermittelten Inhalte, so dass das Lernen signifikant wird, was eine ständige Übung kritischer Reflexion erfordert.

[...] das propositionierte theoretische Wissen wird daher zum Wissen der Praxis artikuliert, gleichzeitig resignierend und von ihnen neu gekennzeichnet. Die Rolle der Theorie besteht darin, lehrern

RC: 96094

Verfügbar in: https://www.nucleodoconhecimento.com.br/bildung- 
Perspektiven von Analysen anzubieten, um das Historische, Soziale, Kulturelle, Organisatorische und sich selbst als Profis, in dem ihre Unterrichtstätigkeit stattfindet, zu verstehen, in sie einzugreifen und sie zu transformieren (GHEDIN, 2012, S. 31).

Es ist notwendig, dass der Professor sich die Theorie aneignet, damit er die Unterrichtspraxis im Einklang mit den Anforderungen der Gesellschaft reflektieren und interpretieren kann, was Veränderungen in der pädagogischen Praxis impliziert, so dass es möglich ist, die Autonomie und Dynamik des Lehrers zu stärken (TREBIEN et al., 2020). Die pädagogische Praxis ist der Ausgangspunkt und der Ausgangspunkt des kontinuierlichen Bildungsprozesses und gleichzeitig ein Weg, der die Transformation des Handelns des Lehrers ermöglicht und somit die theoretische Grundlage von Subventionen sucht, die die Stärkung der Praxis auf reflexive, dialogische und interaktive Weise ermöglichen (GHEDIN, 2012). Es liegt daher am Lehrer, innovationsbereit zu sein, damit er den Anforderungen der Gesellschaft im Bereich des Unterrichts gerecht werden kann, damit er die Praxis und die berufliche Praxis verändern kann (RODRIGUES; LIMA; VIANA, 2017).

Von dem Moment an, in dem man beginnt, über Weiterbildung nachzudenken und wie sie sich vielfältigen Fortschritten (auch einer technologischen Ordnung) anpassen muss, ist ein bewusstes Bewusstsein für die soziale Qualität der Bildung und der schulpädagogischen Praxis erforderlich (JUNGES; KETZER; OLIVEIRA , 2018). Es versteht sich daher, dass eine erkenntnistheoretische und gesellschaftliche Qualitätserziehung ein grundlegendes Ziel verfolgt: Die Notwendigkeit einer kreativen und strategischen pädagogischen Praxis wird verteidigt und darf daher nicht durch die einfache Wiederholung bestehender Praktiken geprägt werden (GHEDIN, 2012). In diesem Sinne ist die Ausarbeitung und Anwendung von auf die Ausbildung ausgerichteten Politiken von wesentlicher Bedeutung. Heutzutage sind diejenigen, die sich auf die Leistung von Technologien im Klassenzimmer konzentrieren, von grundlegender Bedeutung. Es ist eine Realität, die einen sorgfältigen Umgang mit Theorie und Praxis in der Lehre erfordert (TREBIEN et al, 2020).

RC: 96094

Verfügbar in: https://www.nucleodoconhecimento.com.br/bildung- 
Es sollte jedoch auf ein Problem aufmerksam gemacht werden, mit dem das brasilianische Bildungssystem konfrontiert ist. In Brasilien haben historisch gesehen die vorherrschenden Modelle der Weiterbildung eine rein instrumentelle Konzeption der Unterrichtsarbeit (FERREIRA; ALBUQUERQUE; LEAL, 2007). In diesem Sinne wird der Lehrer dazu gebracht, Techniken zu reproduzieren und führt passiv die bereits fertigen Vorschläge aus. Es wird festgestellt, dass die Normen in vielen Fällen ein Lehrmodell vorschreiben, das den Schüler zu einem aktiven, partizipativen und autonomen Lernen führt. In der Praxis gibt es jedoch keine Artikulation dieses pädagogischen Wissens in Weiterbildungskursen (TREBIEN et al., 2020). Die Weiterbildung trägt jedoch zur Verbesserung des Lehrers und damit zur Qualität des Unterrichts bei. So werden mehrere Kenntnisse der pädagogischen Praxis für die Resignation der Unterrichtspraxis notwendig, was eine ständige Suche und ein ständiges Lernen seitens des Lehrers impliziert.

\section{LEHRDISTANZ ALS INSTRUMENT DER WEITERBILDUNG}

Der Einsatz von Technologien und Fernunterricht ist eine Realität, die von den Lehrern berücksichtigt werden muss, da die Pandemie eine Art Bruch der Beziehungen und folglich der Bildung verursacht hat. Es wird geschätzt, dass sich die Schule selbst mit der Kontrolle von COVID an eine andere Realität anpassen sollte, wenn nicht sogar vollständig an den Online-Unterricht,zumindest an die Übernahme durch hybride Modelle (LENZI et al., 2021). In Bezug auf die Schwierigkeiten des Online-Unterrichts betrachteten Gonçalves et al. (2020) die Beziehung zwischen der Schule und der Familie als grundlegend, da es dem Schüler überlassen bleibt, die Entwicklung des Schülers in seinem Zuhause zu verfolgen. Nicola und Paniz (2016) fügen hinzu, dass es unabhängig von der gewählten Technologie wichtig ist, dass der Lehrer im Hinterkopf bedenkt, dass die Diversifizierung der pädagogischen technologischen Ressourcen für den Erfolg des Lernunterrichts unerlässlich sein wird.

RC: 96094

Verfügbar in: https://www.nucleodoconhecimento.com.br/bildung- 
Darüber hinaus ist es notwendig, Umgebungen zu suchen, die die tägliche Realität und die Bedürfnisse der Gesellschaft, in die der Schüler eingefügt ist, übersetzen (ARAÚJO et al. 2011).

[..] Das "Klassenzimmer", übersetzt in einen virtuellen Raum, in dem unterrichtsreich zur Verfügung gestellt wird, reicht nicht aus, um das gewünschte Ergebnis zu gewährleisten. Wie in einer Schule ist es auf die traditionellste Weise notwendig, alle beteiligten Agenten zu berücksichtigen. Es sollte auch gesagt werden, dass, selbst wenn man eine Vorstellung hat, die näher an der Realität ist und dass man das Ideal der Teilnehmer des Prozesses hat, dies nicht ausreicht, um die Erreichung der vorgeschlagenen Bildungsziele zu sichern. Mit anderen Worten, es geht nicht darum, die Komponenten einer Schule zu haben und sie an derselben Stelle zusammenzustellen. Was mit dem Konzept der Schule gewünscht wird, ist die harmonische Integration der Teile, so dass die Bedienung dieses Getriebes möglichst viele Kanten aufzeigt (SOARES FILHO, 2013, S.418).

Olivetti und Periotto (2014) kamen bei der Befragung von 88 Lehrern zu dem Schluss, dass die Weiterbildung für die Wirksamkeit des Einsatzes von Technologien im Biologieunterricht von grundlegender Bedeutung ist, da die Biologie allein schon immer andere als theoretische Ressourcen erfordert hat, wie z.B.: Labore und Feld. Darüber hinaus sollte der Lehrer bedenken, dass der Biologieunterricht ein Werkzeug für die kritische und soziale Konstruktion des Faches ist und es daher notwendig ist, alle Ressourcen zu nutzen, damit der Schüler eine bessere Leistung hat (LEITE et al., 2017), einschließlich der Übernahme des Bewusstseins, dass der Student Biologie in seiner täglichen Realität verstehen muss (DURÉ et al., 2018).

Den Biologieunterricht überdenken, Brandim und Nogueira (2018); Machado (2017) führte eine Reflexion über die angewandten Praktiken und die Möglichkeiten technologischer Werkzeuge im Biologieunterricht durch und betonte die Notwendigkeit für den Lehrer, eine ständige Ausbildung zu suchen, um das Ergebnis seiner beruflichen Praxis und seiner sozialen Rolle als Lehrer zu verbessern. Zusätzlich zu dem Kontext des Lehrlernens, das sich auf das unterrichtete Fach konzentriert, sollte der zeitgenössische Lehrer bedenken, dass er auch ein technologischer Pädagoge wird, das heißt, der dafür verantwortlich ist, dem Schüler

RC: 96094

Verfügbar in: https://www.nucleodoconhecimento.com.br/bildung- 
den Einsatz von Technologie auf konstruktivistische Weise beizubringen, wobei die besten Optionen für die Konstruktion von kritischem Denken und eine bessere Aufnahme von Inhalten aufgezeigt werden, die in ihrem Formierungsprozess dienen können. und damit in das digitale Verhalten eingreifen (ROCHA, 2013).

Die Virtualisierung von Bildungssystemen setzt die Veränderung der aktuellen Unterrichtsmodelle und Unterrichtspraktiken voraus, und so "zwingt" die Situation den Lehrer, neue Rollen zu übernehmen und so auf Wege zu kommunizieren, an die er nicht gewöhnt war (CARRARI; SOUZA; BEHR, 2017). Der Lehrer ist nicht nur für die Weitergabe von Wissen an seine Schüler verantwortlich, denn in diesem Moment muss er den Lernprozess des Schülers leiten, damit er seine Fähigkeiten entwickeln kann, das heißt, ihm muss geholfen werden, zu lernen und somit seine Autonomie zu entwickeln (ROSALIN; CRUZ; MATTOS, 2017). Der Lehrer muss begleiten, motivieren, dialogieren, ein Führer, Vermittler sein, eine positive menschliche Interaktion fördern und vermitteln (GOULÃO, 2012). Es wird erwartet, dass es ein Moderator in zwischenmenschlichen und intrapersonalen Beziehungen sein wird und seine Leistung kontinuierlich selbst bewertet und Praktiken überprüft.

Die Einführung von Technologien in die Unterrichtsumgebung ist eine Möglichkeit, die Schüler zu stimulieren und ihre Emotionen, Zuneigungen und Einstellungen zu lenken (DIAS, 2008). Der Lehrer muss in diesem neuen technologischen Szenario Funktionen übernehmen, die die Schaffung und Nutzung digitaler Ressourcen wecken, was bedeutet, dass er seine Unterrichtsstrategien überprüft und die Interaktionen durch Technologie verbessert. Für diese Dynamik ist es notwendig, die Besonderheiten der Kanäle zu verstehen, die synchrone und asynchrone OnlineKommunikation ermöglichen (SALMON, 2000). Es bedarf einer guten Kommunikationsstruktur, um eine authentische virtuelle Lerngemeinschaft zu generieren, damit der Schüler vernetzt und motiviert ist. Regelmäßige Kommunikation mit den Schülern muss von verschiedenen Kommunikationskanälen

RC: 96094

Verfügbar in: https://www.nucleodoconhecimento.com.br/bildung- 
aus erfolgen, damit sie erkennen, dass die Anwesenheit des Lehrers unerlässlich ist. Das "leere" virtuelle Klassenzimmer muss resigniert werden (GOULÃO, 2012).

Das leblose Klassenzimmer ohne soziale und kognitive Präsenz ist ein "lebloser" Raum (MOREIRA; FERREIRA; ALMEIDA, 2013). Virtuelle asynchrone Kommunikationsklassenzimmer werden als "Foren" bezeichnet. Sie sind grundlegend und entsprechen dem Wesen der digitalen vernetzten Bildung, da sie keinen zusammenfluss von Lehrern und Schülern in Raum und Zeit erfordern und somit eine größere Flexibilität in diesem Bildungsmodell besteht (MOREIRA; HENRIQUES; BARROS, 2020). Damit diese Klassenzimmer einem leistungsfähigen Kommunikationsinstrument entsprechen, ist es entscheidend, dass sie gut organisiert und strukturiert sind, was erfordert, dass die Gemeinschaft ihre Betriebsregeln kennt und befolgt, damit virtuell-digital sinnvolles Lernen fördert (SOUZA; ARAGON, 2018). Es wird empfohlen, dass in dieser virtuellen Umgebung für jede Aktivität Leerzeichen vorhanden sind.

Ein Raum für die Kommunikation von Nachrichten und Warnungen, ein weiterer für die Beantwortung der Zweifel der Schüler, ein weiterer informeller Raum, der eine entspanntere Interaktion zwischen Schülern und Lehrern ermöglicht, und Räume, die für jede Art von Aktivität geschaffen werden, sind notwendige Strategien für die Effizienz des Digitalen (MOREIRA; HENRIQUES; BARROS, 2020). Es gibt eine Reihe von Vorteilen, die durch digitale Technologien gefördert werden, aber die Weiterbildung, d.b. die Fortsetzung, ist von entscheidender Bedeutung, da in diesem Bereich ständig Innovation und Integration neuer Techniken stattfinden, was erhebliche Veränderungen in der Unterrichtspraxis erfordert (MOREIRA; HENRIQUES; BARROS, 2020). In diesem Sinne ist es notwendig, die Software zu kennen, zu verstehen, was mit ihrer Verwendung aus pädagogischer Sicht beabsichtigt ist, und $\mathrm{zu}$ verstehen, ob diese spezifische digitale Ressource am besten geeignet ist (CARRARI; Souza, SOUZA, BEHR, 2017).

RC: 96094

Verfügbar in: https://www.nucleodoconhecimento.com.br/bildung- 
Denn die einfache Nutzung digitaler Schnittstellen allein garantiert noch keine Fortschritte oder Innovationen in der Bildungspraxis (SOUZA; ARAGON, 2018). Mehrere Social Web-Schnittstellen werden nicht ausreichend genutzt, wenn die übernommenen Verweise immer noch die im Web 1.0 erworbenen Praktiken replizieren. Aus diesen Gründen und darüber hinaus ist es notwendig, aktive und konstruktive pädagogische und didaktische Praktiken zu fördern (ROSALIN; CRUZ; MATTOS, 2017). Wissen und Lernen müssen kollaborativ entwickelt werden. Netzwerkbildung ist aufgrund ihres eigenen Wesens ein Prozess, der die tiefe Einbeziehung verschiedener an dieser Artikulation beteiligter Akteure erfordert, insbesondere die Definition der Ziele und Lernpfade der Gemeinschaft.

Der Prozess hat auch Auswirkungen auf die engen Beziehungen, die kollaborativ zwischen den Peers aufgebaut werden, die die Prozesse der Innovation und der Schaffung von Wissen aus digitalen Pfaden unterstützen (MOREIRA; HENRIQUES; BARROS, 2020). Für den kollektiven Aufbau dieses neuen Wissens ist es immer häufiger und schneller geworden, dass die Nutzung von Offene Bildungsressourcen (OER) wächst und erweitert wird. Diese haben den Zugang und die freie Nutzung von Inhalten und Technologien gefördert (GOULÃO, 2012). Solche Ressourcen entsprechen einer unerschöpflichen Quelle digitaler Ressourcen, die mit Aktivitäten verknüpft werden können, die auf die Präsentation und Anreicherung von Inhalten abzielen. Es gibt Plattformen, die verschiedene Formen der Herangehensweise an Wissen ermöglichen (Plattformen, die Video, Audio und Bild integrieren, wie YouTube und andere soziale und digitale Netzwerke / Medien) (SOUZA; ARAGON, 2018).

\section{WISSEN, DAS FÜR DEN ONLINE-UNTERRICHT BENÖTIGT WIRD}

Es ist nicht das Privileg der Biowissenschaften, sich Herausforderungen im Lehrprozess des Lernens zu stellen, oder noch mehr als das Lehren und wie man lehrt. Im Kontext des Online-Unterrichts wurden diese Herausforderungen nur noch

RC: 96094

Verfügbar in: https://www.nucleodoconhecimento.com.br/bildung- 
erweitert, indem die unterentwickelten technologischen Fähigkeiten des Lehrers gefordert wurden. Duré et al. (2018) weisen darauf hin, dass die größte Herausforderung für Lehrer sowohl im Präsenzunterricht als auch im OnlineUnterricht darin besteht, die Schüler für den Unterricht zu interessieren, so dass der Unterrichtsprozess bei der Suche nach Ansätzen, die ihre persönlichen und sozialen Bedürfnisse berücksichtigen, tendenziell effektiver ist. Eine weitere Strategie, die von Lehrern in diesem Prozess übernommen werden sollte, ist die breite Diversifizierung der Unterrichtsmechanismen, wie Videos auf YouTube, Filme, Spiele, gesucht (OLIVETTI und PEIOTTO, 2014; NICOLA und PANIZ, 2017; ARAÚJO et al., 2011; LEITE et al., 2017; LEÃO et al., 2018)

Tabelle 1- Vermittlung von Wissen und Indikationen für Online-Bildung

Wissen, das für den Online-Biologieunterricht benötigt wird Autoren

Passen Sie den Unterricht an die Realität des Schülers Araújo et al. 2011; an, ob sozial oder persönlich Leite et al., 2017; Machado, 2017; Leão et al., 2018.

Suchen Sie nach Videos, Spielen, Apps, die Dynamik in Araújo et al., 2011; den Biologieunterricht bringen können Leão et al., 2018.

Suchen Sie nach Bewertungsmethoden, die den Araújo et al., 2011; Schüler einbeziehen, einschließlich Gruppenmethodik, Leite et al., 2017; z. B. erstellen von Ausstellungsvideos Leão et al., 2018.

Konzeptionelle Karte Leão et al., 2018

Verwendung von Quiz (Instagram) Souza et al., 2020

Recherche in verschiedenen Online-Medien zu den Machado, 2017; exponierten Themen. Die Gelegenheit nutzen, Brandim und kritisches Denken und Analyse einiger Positionen zu Nogueira, 2018 diesem Zeitpunkt zu entwickeln

Diversifizierung von Youtube-Ressourcen, Filmen, Olivetti und Peiotto,

RC: 96094

Verfügbar in: https://www.nucleodoconhecimento.com.br/bildung- 
Spielen, Texten

Anpassung von Sprache, Licht und Aufnahmeort
2014; Nicola und

Paniz, 2017.

Soares Filho, 2013.

Quelle: Autor

Souza et al. (2020) führten eine Lehrerfahrung durch das "Quiz" -Tool auf Instagram durch, als eine Art Wissenstest. Die Ergebnisse zeigten, dass sich die Studenten motiviert und aufgeregt fühlten, einschließlich der Entwicklung einer Wettbewerbsbewegung untereinander. Bewertet man, wie die Schüler über dieses Tool dachten, war das Ergebnis auch positiv, weil die Schüler involviert und motiviert waren. Schließlich wird die Sorgfalt des Lehrers beim Online-Unterricht, der Versuch, seine Sprache im Kontext der Schüler zu aktualisieren und ihre technologischen Ressourcen wie Licht und Ton zu untersuchen, sicherlich als Strategie für den Erfolg des Unterrichts durch bessere Schülerunterstützung gemacht (SOARES FILHO, 2013).

Die Gemeinsame nationale Lehrplanbasis (BNCC) weist auf die Notwendigkeit gemeinsamer Anstrengungen zwischen Lehrern, Forschern und nationalen Organisationen hin, damit wesentliche Kompetenzen für brasilianische Grundschüler entwickelt werden, einschließlich derjenigen, die sich auf die digitale Umgebung beziehen (FERREIRA et al., 2021). Diese müssen so entwickelt werden, dass die täglichen Anforderungen erfüllt werden. BNCC präsentiert zehn Kompetenzen, die auf menschliches und integrales Training abzielen, einschließlich "digitaler Kultur". Daher befasst es sich mit der Entwicklung von Fähigkeiten und Fertigkeiten im Zusammenhang mit dem Einsatz digitaler Technologien im Klassenzimmer, und diese sollten für verschiedene soziale Praktiken geeignet sein (MATTAR et al., 2020). Daher muss die Weiterbildung den Lehrkräften helfen, Informations- und Kommunikationstechnologien zu verstehen, zu nutzen und zu schaffen (CHIOSSI; COSTA, 2018)

RC: 96094

Verfügbar in: https://www.nucleodoconhecimento.com.br/bildung- 
Der Einsatz digitaler Technologien sollte kritisch, signifikant, reflexiv und ethisch erfolgen und tägliche Praktiken im Bildungskontext einführen. Ziel ist es, Kommunikation, Zugang, Verbreitung von Informationen, Wissensproduktion, Problemlösung, die Ausübung von Protagonismus und Autorschaft im Prozess der Interaktion mit solchen Technologien im Bildungskontext zu fördern (LEITE; SILVA, 2017). In diesem Sinne schlägt der BNCC durch das Verständnis der Einbeziehung digitaler Technologien in die Konstruktion des Lehr-Lern-Prozesses und in die Lösung alltäglicher Probleme vor, dass Fachleute geschult werden sollten, um digitales in den Klassenzimmern einführen zu können (PIFFERO et al., 2020). Lehrkräfte unterschiedlicher Bildungsstufen und mehrerer Fächer sollten Fähigkeiten und Fertigkeiten entwickeln, die diese Inklusion ermöglichen (CHIOSSI; COSTA, 2018).

Daher sollten nationale Programme und Politiken, die die Einbeziehung von Technologien in die Bildung beinhalten, Strategien fördern, die einen effektiven Zugang zu digitalen Technologien ermöglichen, aber es gibt immer noch zahlreiche Barrieren und Herausforderungen (LEITE; SILVA, 2017). Informations- und Kommunikationstechnologien müssen über die technische und instrumentelle Nutzung des Digitalen hinausgehen (PIFFERO et al., 2020). Es gibt zwei Faktoren, die den Zugang zum Digitalen im Klassenzimmer verzögern können: physische und strukturelle Bedingungen und unzureichende Ausbildung (FERREIRA et al., 2021). In Bezug auf die Strukturierung der Schulen und die Lehrerausbildung selbst kann das 1997 ins Leben gerufene Nationales Programm für Bildungstechnologie (PROINFO) hervorgehoben werden. Dies hat neue Entwicklungen ausgelöst und das Programm für Innovation und vernetzte Bildung erreicht. Diese Initiativen werden zu anderen hinzugefügt, die seit den 1970er Jahren entwickelt wurden.

Ziel ist es, den Schulen eine rechnerische Struktur zur Verfügung zu stellen, so dass eine angemessene Lehrerqualifikation gefördert werden kann, die den Einsatz von TDIC im Bildungskontext ermöglicht (FERREIRA et al., 2021). Um eine konkrete

RC: 96094

Verfügbar in: https://www.nucleodoconhecimento.com.br/bildung- 
Politik im Bereich der Informationstechnologie zu etablieren, um die Wirtschaft des Landes zu nutzen, sind Technologien Teil der unterschiedlichsten Kontexte geworden, wurden jedoch noch nicht effizient eingeführt, da es immer noch schwierig ist, eine digitale Kultur im Unterricht zu implementieren (CHIOSSI; COSTA, 2018). Mit der Notwendigkeit der Einführung von Fernunterricht zeigten sich jedoch einige Ungleichheiten aufgrund der Pandemie (SARAIVA; TRAVERSINI; LOCKMANN, 2020). So sehr einige Schulen Momente des digitalen Treffens von den verschiedenen Plattformen aus fördern können, ist der Zugang immer noch ein Problem.

Darüber hinaus erschwert die mangelnde Beherrschung der Nutzung von Plattformen, Netzwerken und digitalen Medien durch den Lehrer auch die Integration von Technologie in die Unterrichtsumgebung. Die Beherrschung bestimmter Werkzeuge und Technologien setzt die Kenntnis solcher Möglichkeiten voraus. Neue Technologien entstehen und werden jeden Tag verbessert, was vom Lehrer eine ständige Suche nach der Verbesserung seiner Fähigkeiten und Fähigkeiten im Zusammenhang mit dem Umgang mit solchen Plattformen und digitalen Tools erfordert (MATTAR et al., 2020). Das für die Unterrichtsübung notwendige Wissen durchdringt einige Bereiche. Sie werden aus bestimmten Kategorien klassifiziert (TARDIF, 2010). Sie sind das Wissen um disziplinäre Ordnung; berufliche Kenntnisse; curriculares Wissen und Wissen im Zusammenhang mit dem experimentellen Feld (MATTAR et al., 2020).

Die technologischen Fähigkeiten, die dem Lehrer in der Unterrichtsübung in Rechnung gestellt werden, sind mit bestimmten Kenntnissen und Fähigkeiten im Zusammenhang mit dem Umgang mit digitalen Netzwerken und Medien verbunden (FERREIRA et al., 2021). In diesem Sinne, um das Lernen sinnvoll und an den Einsatz von Technologie gebunden zu machen, werden einige Fähigkeiten von größter Bedeutung, aber damit sie ausgeführt werden können, ist es notwendig, dass der Lehrer über das notwendige Wissen über ihre Nutzungsmöglichkeiten verfügt

RC: 96094

Verfügbar in: https://www.nucleodoconhecimento.com.br/bildung- 
(PIFFERO et al., 2020). Die Beherrschung von Computerkenntnissen, Software, von Werkzeugen, Medien und verschiedenen virtuellen Umgebungen (wie Google Meet und anderen Plattformen) erfordert eine gewisse Alphabetisierung und Geläufigkeit der digitalen Sprache (CHIOSSI; KÜSTE, 2018). Schulungen sollten auf dieser Betonung basieren, damit der Unterricht im virtuellen Umfeld ein effizientes, dynamisches und attraktives Lernen für die Studierenden fördert.

\section{ENDGÜLTIGE ÜBERLEGUNGEN}

Basierend auf der Annahme, einige Hinweise auf das Wissen des Lehrers in Bezug auf den Online-Unterricht zu geben, basierte diese Studie auf der explorativen Methodik und suchte nach Anspielungen und Indikationen innerhalb anderer bereits durchgeführter Studien. Um die abschließenden Überlegungen dieser Studie zu verdeutlichen, wurde die Leitfrage beantwortet: Wie können sich Life-SciencesLehrer vorbereiten und welche grundlegenden Werkzeuge sollten sie kennen, um ihren Online-Unterricht vorzubereiten? Die Studie zeigte, dass Gymnasial- und Grundschullehrer die Notwendigkeit haben, mehrere werkzeuge und Strategien zu verwenden, um ihren Online-Unterricht vorzubereiten. Es reicht nicht aus, nur Ausstellungsunterricht zu halten, es ist notwendig, die Schüler einzubeziehen. Die Interaktion mit solchen Technologien muss jedoch angeregt werden, damit das Lernen sinnvoll ist, ist es notwendig, bestimmte Techniken, Plattformen, Werkzeuge usw. zu beherrschen und zu kennen.

Einige effektive Werkzeuge, auf die in der Literatur hingewiesen wird, sind: Konzeptionelle Karten, YouTube-Videos, Filme, Spiele, Google-Suche und andere Netzwerke, kurz gesagt, Werkzeuge, die sich beim Schüler entwickeln, nicht nur im Kontext der Biologie, sondern auch, die den bewussten und effektiven Einsatz von Technologien fördern. Darüber hinaus ist eine gewisse technische Sorgfalt von grundlegender Bedeutung, wie zum Beispiel: Angemessenheit der Sprache für die Klasse, unter Berücksichtigung ihrer Altersgruppe, Licht und Audio. Aufgrund des

RC: 96094

Verfügbar in: https://www.nucleodoconhecimento.com.br/bildung- 
Pandemieszenarios und folglich des Mangels an Klassen im Klassenzimmer und den Möglichkeiten, auf das Feld zu gehen, brachte diese Studie einige Einschränkungen mit sich, die durch Forschung mit Lehrern und Schülern besser untersucht werden konnten. Es wird darauf hingewiesen, dass weitere Studien in angewandter Weise mit Schülern und Lehrern durchgeführt werden können, um Strategien und Werkzeuge zu untersuchen, die sich für den Unterricht von Online-Biologie in der Ober- und Grundschule als wirksam erweisen.

\section{VERWEISE}

ARAÚJO, Magnólia Fernandes; SOUSA, Rute Alves; SOUSA, Ivanise Cortez de. Instrumentalização para o Ensino da Biologia. 2 ed. EDFRN, 2011.

BRANDIM, Maria Rejane Lima; NOGUEIRA, Johnson Fernandes. Ensino de Ciências e de Biologia: reflexões e práticas- Parnaíba: Edufpi, 2018.

CARRARO, W. B. W. H.; SOUZA, M.; BEHR, A. Ferramentas de educação a distância utilizadas por profissionais de contabilidade visando a educação continuada. Revista EDaPECl, v. 17, n. 2, p. 144-160, 2017.

CHIOSSI, R. R.; COSTA, C. S. Novas formas de aprender e ensinar: a integração das tecnologias de informação e comunicação (TIC) na formação de professores da educação básica. Texto Livre: Linguagem e Tecnologia, v. 11, n. 2, p. 160-176, 2018.

DIAS, P. Da e-moderação à mediação colaborativa nas comunidades de aprendizagem. Educação, Formação e Tecnologias, v. 1, n. 1, p. 4-10, 2008.

DURÉ, Ravi Cajú; et. al; Ensino De Biologia E Contextualização Do Conteúdo: Quais Temas O Aluno De Ensino Médio Relaciona Com O Seu Cotidiano? Experiências em Ensino de Ciências V.13, No.1, 2018.

RC: 96094

Verfügbar in: https://www.nucleodoconhecimento.com.br/bildung- 
FALSARELLA, A. M. Formação continuada e prática de sala de aula: os efeitos da formação continuada na atuação do professor. Campinas, São Paulo: Autores Associados, 2004.

FERREIRA, L. P. S. et al. Autopercepção das competências digitais pelos professores da educação básica sergipana durante o ensino remoto. Scientia Plena Jovem, v. 8, n. 1, 2021.

FERREIRA, T. B.; ALBUQUERQUE, E. B. C.; LEAL, T. F. Formação Continuada de Professores: questões para reflexão. Belo Horizonte: Autêntica, 2007.

GADOTTI, M. Boniteza de um sonho: Ensinar e Aprender com sentido. São Paulo: Editora e Livraria Instituto Paulo Freire, 2008.

GHEDIN, E. Professor Reflexivo: da alienação da técnica à autonomia da crítica. In: PIMENTA, S. G.; GHEDIN, E (Orgs.). Professor reflexivo no Brasil: gênese e crítica de um conceito. $7^{\mathfrak{a}}$ ed. São Paulo: Cortez, 2012.

GIL, Antonio Carlos Gil. Método de Pesquisa. Ed. Atlas, São Paulo, 2018.

GONÇALVES, Janaína Oliveira. MIRANDA, Maurício Fernando Oliveira de. GONÇALVES JÚNIOR, Edson. Uma reflexão sobre a parceria da família e escola em tempos de COVID-19: Aspectos pedagógicos, econômicos e jurídicos. Revista Científica Multidisciplinar Núcleo do Conhecimento. Ano 05, Ed. 11, Vol. 06, pp. 141 154. Novembro de 2020. ISSN: 2448-0959, Link de acesso: https://www.nucleodoconhecimento.com.br/educacao/economicos-e-juridicos, DOI: 10.32749/nucleodoconhecimento.com.br/educacao/economicos-e-juridicos

GOULÃO, M. F. The use of Forums and collaborative learning: $A$ study case. Procedia - Social and Behavioral Sciences, n. 46, p. 672-677, 2012.

RC: 96094

Verfügbar in: https://www.nucleodoconhecimento.com.br/bildungde/biowissenschaften 
JUNGES, F. C.; KETZER, C. M.; OLIVEIRA, V. M. A. de. Formação continuada de professores: saberes ressignificados e práticas docentes transformadas. Educação \& Formação, v. 3, n. 9, p. 88-101, 2018.

LEÃO, Marcelo Franco; et al; Estratégias didáticas voltadas ao ensino das Ciências. Ed. Edibrás. Uberlândia, 2018.

LEITE, M. S.; SILVA, S. R. F. da. Redimensionamento da Computação em Processo de Ensino na Educação Básica: O pensamento Computacional, o Universo e a Cultura Digital. In: VI Congresso Brasileiro de Informática na Educação, 2017.

LEITE, Paula Rayanny Mendonça; et al; O ensino da biologia como uma ferramenta social, crítica e educacional. RECH- Revista Ensino de Ciências e Humanidades Cidadania, Diversidade e Bem-estar. ISSN 2594-8806 - 400. Ano 1, Vol 1, Número 1, Jul-Dez, 2017, p. 400-413

LENZI, Juliana Fernandes de Almeida Castro. SANTOS, Marijara Barbosa Bragato. Sociedade Pós-Moderna Frente À Covid-19: Educadores Em Ação. Revista Científica Multidisciplinar Núcleo do Conhecimento. Ano 06, Ed. 03, Vol. 11, pp. 100 111. Março de 2021. ISSN: 2448-0959, Link de acesso: https://www.nucleodoconhecimento.com.br/educacao/educadores-em-acao

MACHADO, Claudia Pinto/ Ensino de ciências [recurso eletrônico]: práticas e exercícios para a sala de aula- Caxias do Sul, RS: Educs, 2017.

MATTAR, J. et al. Competências e funções dos tutores online em educação a distância. Educação em Revista, v. 36, 2020.

MOREIRA, J. A.; FERREIRA, A. G.; ALMEIDA, A. C. Comparing communities of inquiry in higher education students: one for all or one for each? OpenPraxis. Internacional Council for Open and Distance Education, v. 5, n. 2, p. 165-178, 2013.

RC: 96094

Verfügbar in: https://www.nucleodoconhecimento.com.br/bildungde/biowissenschaften 
MOREIRA, J. A.; HENRIQUES, S.; BARROS, D. M. V. Transitando de um ensino remoto emergencial para uma educação digital em rede, em tempos de pandemia. Dialogia, n. 34, p. 351-364, 2020.

NICOLA, Jéssica Anese; PANIZ, Catiane Mazocco. A importância da utilização de diferentes recursos didáticos no ensino de biologia. Infor, Inov. Form., Rev. NEaDUnesp, São Paulo, v. 2, n. 1, p.355-381, 2016. ISSN 2525-3476.

OLIVETTI, Maria Madalena de Carvalho; PERIOTTO, Fernando. biologia e as novas tecnologias educacionais, um foco para a educação contemporânea. Revista Eletrônica Científica Inovação e Tecnologia Universidade Tecnológica Federal do Paraná Câmpus Medianeira. Vol.1, p. 9- 2014.

PIFFERO, E. de. L. F. et al. Metodologias Ativas e o ensino de Biologia: desafios e possibilidades no novo Ensino Médio. Ensino \& Pesquisa, v. 18, n. 2, p. 48-63, 2020

ROCHA, Jéssica. A percepção da ciência pelos alunos do curso de pedagogia UAB/UFMG. V Seminário Internacional de Educação a Distância. UFMG, 2013.

RODRIGUES, P. M. L.; LIMA, W. dos. S. R.; VIANA, M. A. P. A importância da formação continuada de professores da educação básica: a arte de ensinar e o fazer cotidiano. Saberes Docentes em Ação, v. 3, n. 1, p. 28-47, 2017.

ROSALIN, B. C. M.; CRUZ, J. A. S.; MATTOS, M. B. G. de. A importância do material didático no ensino a distância. Revista on line de Política e Gestão Educacional, v. 21, n. 1, p. 814-830, 2017.

SALMON, G. E-Moderating. The Key to Teaching and Learning Online. London: Kogan Page, 2000.

RC: 96094

Verfügbar in: https://www.nucleodoconhecimento.com.br/bildung- 
SARAIVA, K.; TRAVERSINI, C. S.; LOCKMANN, K. A educação emtempos de COVID-19: ensino remoto e exaustão docente. Práxis educativa, v. 15, p. 1-24, 2020.

SOARES FILHO, Daniel. Conteúdos EAD: Pequenos detalhes que fazem a diferença. V Seminário Internacional de Educação a Distância. UFMG, 2013.

SOUZA, Dominique Guimarães; et. al. Redes sociais e o ensino de biologia: O uso do Quis do Instagram como Recurso Didático. Recite - Revista Carioca de Ciência Tecnologia e Educação · December 2020.

SOUZA, S. S. dos. S. de.; ARAGON, G. T. Estilos de aprendizagem e ensino a distância na perspectiva da inclusão. EaD em Foco, v. 8, n. 1, 2018.

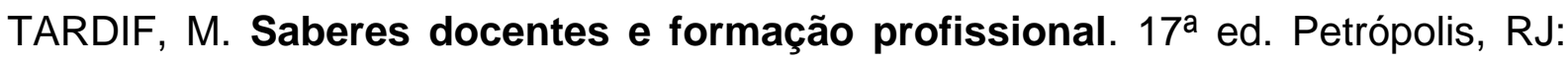
Vozes, 2010.

TREBIEN, M. M et al. Formação continuada de professores: uma epistemologia da prática. Ambiente: Gestão e Desenvolvimento, v. 13, n. 1, p. 91-102, 2020.

Eingereicht: September 2021.

Genehmigt: September 2021.

RC: 96094

Verfügbar in: https://www.nucleodoconhecimento.com.br/bildung- 